\title{
Impact of socioeconomic status on survival of colorectal cancer patients
}

\author{
Qian Zhang ${ }^{1,2}$, Yufu Wang ${ }^{1,2}$, Hanqing Hu${ }^{1,2}$, Rui Huang ${ }^{1,2}$, Lei Xie ${ }^{1,2}$, Enrui Liu ${ }^{1,2}$, \\ Ying-Gang Chen ${ }^{1,2}$, Guiyu Wang ${ }^{1,2}$ and Xishan Wang ${ }^{1,2,3}$ \\ ${ }^{1}$ Department of Colorectal Surgery, The Second Affiliated Hospital of Harbin Medical University, Harbin, Heilongjiang 150086, \\ PR China \\ ${ }^{2}$ Colorectal Cancer Institute of Harbin Medical University, Harbin, Heilongjiang 150086, PR China \\ ${ }^{3}$ Department of Colorectal Surgery, Cancer Hospital of Chinese Academy Sciences, Beijing, 100021, PR China
}

Correspondence to: Xishan Wang, email: wxshan1208@126.com

Guiyu Wang, email: guiywang@163.com

Ying-Gang Chen, email: chygang777@126.com

Keywords: socioeconomic, SEER, colorectal cancer, survival, adverse

Received: October 17, 2016 Accepted: May 29, $2017 \quad$ Published: September 13, 2017

Copyright: Zhang et al. This is an open-access article distributed under the terms of the Creative Commons Attribution License 3.0 (CC BY 3.0), which permits unrestricted use, distribution, and reproduction in any medium, provided the original author and source are credited.

\section{ABSTRACT}

Socioeconomic status (SES) has an impact on the survival of various cancers, but it has not been fully understood in colorectal cancer (CRC).The Surveillance, Epidemiology and End Results database was adopted to detect the role of SES in the survival outcomes of CRC. A total of 184,322 eligible patients were included and SES status was analyzed. The multivariable analysis showed that Non-Hispanic Black (HR, 1.20; 95\% CI, 1.15-1.24), being widowed (HR, 1.04; 95\% CI, 1.01-1.07), any Medicaid (HR, 1.36; 95\% CI, 1.33-1.39) and the lowest education level group patients had relative poorer prognosis. Besides, sex, tumor location, age, differentiation level and American Joint Committee on Cancer stage also had significant effects on overall survival of CRC. The individuals were further divided into five groups according to the number of survival-adverse factors. All of the four groups containing adverse factors showed impaired survival outcomes compared with the group containing no adverse factor.

\section{INTRODUCTION}

Colorectal cancer (CRC) is the third most common malignancy in the United States, with 135,430 new cases and 50,260 deaths expected in 2017 [1]. The survival of patients with CRC have improved substantially due to the development of operation skills, chemotherapy drugs, immune treatment and early screening methods including timely colonoscopy examination, blood and fecal test and CT scan, etc. However, the survival rate of patients with CRC has changed little over the past 10 years.

The outcome of CRC is determined by various factors, including American Joint Committee on Cancer AJCC staging, tumor location, and neoadjuvant/adjuvant chemotherapy. Besides, socioeconomic status (SES) is also an important prognosis factor of CRC. Previous data indicated that married individuals possess better prognosis for many major causes of death compared with those who are single, separated, widowed, or divorced [2-4]. In consider of high complexity, length of duration, high cost of cancer-related therapies and the complicated role of SES in CRC survival, we hypothesized that SES factors, including insurance status, marital status, income, and educational level, may contribute to the overall survival (OS) observed in patients with colorectal cancer.

In this study, we used data from the Surveillance, Epidemiology and End Results (SEER) diagnosed between 2007 and 2013 to investigate the impact of SES on OS of CRC in detail.

\section{RESULTS}

Patient baseline characteristics

A total of 184,322 eligible patients were included, including 98,802 (53.6\%) male and 85,520 (46.4\%) 
female patients. The median age was 67 years old with the range 20-108 years old. The median followup time was 24 months. Among them, 15436 (8.4\%) had well differentiation, $113682(61.7 \%)$ moderate differentiation, 26670 (14.5\%) poor differentiation, 3661 (2.0\%) un-differentiation and 24839 (13.5\%) unknown differentiation. The tumor site distribution was as follows: $128342(69.6 \%)$ colon (with 38912 right colon and 14762 left colon), 48676 (26.4\%) rectum and 6157 (3.3\%) large intestine, NOS (not specified). Among these individuals, $43788(23.8 \%)$ were stage I, $44223(24.0 \%)$ stage II, $45857(24.9 \%)$ stage III and $34637(18.8 \%)$ were stage IV. We also analyzed the race distribution: 19108 (10.4\%) were Hispanic, 126482 (68.6\%) Non-Hispanic White, 21201 (11.5\%) Non-Hispanic Black, 1121 (0.6\%) NonHispanic American/Alaska native, and 15767 (8.6\%) were Non-Hispanic Asian or Pacific Islander. Most of the included patients were married (104562, 56.7\%), and single, widowed, and divorced patients were 29172 (15.8\%), $32370(17.6 \%)$ and $18218(9.9 \%)$ respectively. The cohort included $155881(84.6 \%)$ insured patients, 22111 (12.0\%) any-Medicaid patients and 6330 (3.4\%) uninsured patients. Moreover, the characteristics were stratified by sex. It showed that there was a dramatic difference between male and female individuals. Other data including SEER registry, histology, income and education level were also listed in Table 1.

\section{Impact of socioeconomic status on survival}

The crude analysis showed that age, tumor site (colon vs rectum, $\mathrm{HR}=0.90,95 \%$ CI $0.88-0.91$; right vs left colon, $\mathrm{HR}=0.95,95 \%$ CI $0.92-0.98)$, differentiation level, AJCC staging, race, SEER registry, marital status, insurance status, income level and education level were all significantly associated with CRC overall survival (Table 2). After adjustment for confound factors, female sex, age, tumor site (rectum vs colon), differentiation level, AJCC stage, race, SEER registry, marital status, insurance status, and education level were still associated with OS of CRC (Figures 1-4 and Table 2).

To validate the impact of SES on CRC survival, we divided these individuals into five groups according to the number of survival-adverse factors. The cumulative effect of SES factors associated with shorter survival in the multivariable analysis (ie, marital status other than married, insurance status other than "insured," race other than Non-Hispanic Black, and residence in a county within the lowest 1 quartile of education) was shown in Figure 5.

After adjusted for SEER registry, age, and sex, the presence of 1 adverse SES factor (HR, 1.27; 95\% CI, 1.25-1.29), 2 adverse SES factors (HR, 1.55; 95\% CI, 1.51-1.59), 3 adverse SES factors (HR, 1.80; 95\% CI, 1.71-1.90), and 4 adverse SES factors (HR, 2.10; 95\% CI, $1.77-2.48)$ were found to be associated with a gradually higher risk of death when compared with individuals with no adverse SES factors (Table 3 ).

Furthermore, we evaluated the effect of female sex, age, differentiation level, tumor site (right colon vs left colon, rectum vs colon) and AJCC staging on survival of colorectal cancer in the five groups. The data showed that sex, age, differentiation level, and AJCC staging still exert significant effect on the survival of CRC patients in these groups except in the four-adverse-factor group.

\section{DISCUSSION}

As far as we know, the current research is the largest study to date that evaluates the impact of SES factors on the survival of CRC patients. Our data show that NonHispanic Black, being single, divorced or widowed, being uninsured or Medicaid, county-level income are related with an increased risk of death after adjusting for confounding factors including sex, age, SEER registry.

Many studies have detected the relationship between marital status and survival of cancers including CRC [5]. In thyroid cancer [6], gastric cancer [2], pancreatic cancer [3] and colorectal cancer [4], unmarried patients showed poorer prognosis compared with married patients. The reason that marital status is associated with cancer survival is complicated. Unmarried patients might tend to be more depressed and anxious and at higher nonadherence with prescribed treatments than married individuals [7, 8]. The negative emotions impair immune system function and thus may increase the mortality [9]. Aging itself is an adverse prognostic factor in CRC as shown in our result and other studies $[10,11]$ considering that aging impaired immune system, increased oxidative stress, shortening of telomeres, accumulation of senescent cells [12]. The widowed group contained a very high proportion of elderly patients (data not shown), which might result in the poor survival in this group.

Our study found that the insured patients have the best overall survival compared with uninsured and Medicaid patients. A recent study showed that Medicaid or uninsured status was associated with decreased diagnosis rates of nonpalpable prostate cancer and increased conservative management [13], thus impaired the survival of patients. CRC screening should begin at 50 years of age as it has been shown that screening adults aged $50-75$ years reduces CRC mortality. Screening tests for CRC include stool-based tests such as fecal immunochemical test and guaiac fecal occult blood test and direct visualization tests including colonoscopy, CT colonoscopy and flexible sigmoidoscopy [14, 15]. Delayed cancer screen may also affect the survival of cancer patients. Uninsured and Medicaid-insured cancer patients have been shown to present with more advanced disease, less often receive cancer-directed therapy and suffer higher rates of mortality than those with private insurance [16]. For CRC, lack 
Table 1: Characteristics of patients with CRC at the time of diagnosis in the US SEER, 2007 to 2012

\begin{tabular}{|c|c|c|c|c|}
\hline Characteristics & Total No. (\%) & Male & Female & $P$ value \\
\hline Sex & $184,322(100.0)$ & $98802(53.6)$ & $85520(46.4 \%)$ & \\
\hline Age $[$ Median(range)], y & $67(20-108)$ & $60(20-107)$ & $69(20-108)$ & $<0.001$ \\
\hline Quartile $1(<56)$ & $42482(23.0)$ & $23196(12.6)$ & $19286(12.4)$ & \\
\hline Quartile $2(56-66)$ & $46869(25.4)$ & $27601(15.0)$ & $19268(10.4)$ & \\
\hline Quartile 3 (67-77) & $48094(26.1)$ & $26799(14.5)$ & $21295(11.6)$ & \\
\hline Quartile $4(>77)$ & $46877(25.4)$ & $21206(11.5)$ & $25671(13.9)$ & \\
\hline Follow-up time [Median(range)], Mo & $24(0-83)$ & $24(0-83)$ & $24(0-83)$ & $<0.001$ \\
\hline Quartile $1(<8)$ & $43716(23.7)$ & $23232(12.6)$ & $20484(11.1)$ & \\
\hline Quartile $2(8-23)$ & $46554(25.3)$ & $25354(13.8)$ & $21200(11.5)$ & \\
\hline Quartile 3 (24-47) & $47009(25.5)$ & $25220(13.7)$ & $21789(11.8)$ & \\
\hline Quartile $4(>47)$ & $47043(25.5)$ & $24996(13.6)$ & $22047(12.0)$ & \\
\hline Differentiation no. (\%) & & & & $<0.001$ \\
\hline Well & $15436(8.4)$ & $8265(4.5)$ & $7171(3.9)$ & \\
\hline Moderate & $113682(61.7)$ & $61733(33.5)$ & $51949(28.2)$ & \\
\hline Poor & $26670(14.5)$ & $13340(7.2)$ & $13330(7.2)$ & \\
\hline Undifferentiation & $3661(2.0)$ & $1739(0.9)$ & $1922(1.0)$ & \\
\hline Pre-b & $916(0.5)$ & $561(0.3)$ & $355(0.2)$ & \\
\hline Unknown & $23923(13.0)$ & $13141(7.1)$ & $10782(5.9)$ & \\
\hline \multicolumn{5}{|l|}{ Site no. $(\%)$} \\
\hline Colon & $128342(69.6)$ & & & \\
\hline Right colon & $38912(21.1)$ & $18345(9.9)$ & $20567(11.2)$ & 0.000 \\
\hline Left colon & $14762(8.0)$ & $8021(5.4)$ & $6741(2.6)$ & \\
\hline Others & $74668(40.5)$ & & & \\
\hline Rectum & $48676(26.4)$ & $28433(15.4)$ & $20243(11.0)$ & \\
\hline Large intestine, NOS & $6157(3.3)$ & $3153(1.7)$ & $3004(1.6)$ & \\
\hline AJCC stage, no. (\%) & & & & 0.000 \\
\hline $\mathrm{I}$ & $43788(23.8)$ & $23629(12.8)$ & $20159(10.9)$ & \\
\hline II & $44223(24.0)$ & $23305(12.6)$ & $20918(11.3)$ & \\
\hline III & $45857(24.9)$ & $24306(13.2)$ & $21551(11.7)$ & \\
\hline IV & $34637(18.8)$ & $19343(10.5)$ & $15294(8.3)$ & \\
\hline Unstaged & $15817(8.6)$ & $8219(4.5)$ & $7598(4.1)$ & \\
\hline Race no. $(\%)$ & & & & 0.000 \\
\hline Hispanic & $19108(10.4)$ & $10696(5.8)$ & $8412(4.6)$ & \\
\hline Non-Hispanic White & $126482(68.6)$ & $67820(36.8)$ & $58662(31.8)$ & \\
\hline Non-Hispanic Black & $21201(11.5)$ & $10861(5.9)$ & $10340(5.6)$ & \\
\hline Non-Hispanic American/Alaska native & $1121(0.6)$ & $602(0.3)$ & $519(0.3)$ & \\
\hline Non-Hispanic Asian or Pacific Islander & $15767(8.6)$ & $8460(4.6)$ & $7307(4.0)$ & \\
\hline Other & $643(0.3)$ & $363(0.2)$ & $280(0.2)$ & \\
\hline Histology no. (\%) & & & & 0.000 \\
\hline Mucinous adenocarcinoma & $12010(6.5 \%)$ & $6084(3.3)$ & $5926(3.2)$ & \\
\hline Signet ring cell carcinoma & $1710(0.9 \%)$ & $923(0.5)$ & $787(0.4)$ & \\
\hline Carcinoid tumor & $6456(3.5 \%)$ & $3211(1.7)$ & $3245(1.8)$ & \\
\hline Other & $164146(89.1 \%)$ & $88584(48.1)$ & $75562(41.0)$ & \\
\hline Registry no. (\%) & & & & 0.000 \\
\hline San Francisco-Oakland SMSA & $9739(5.3)$ & $5057(2.7)$ & $4682(2.5)$ & \\
\hline Connecticut & $8418(4.6)$ & $4400(2.4)$ & $4018(2.2)$ & \\
\hline Detroit (Metropolitan) & $9386(5.1)$ & $4819(2.6)$ & $4567(2.5)$ & \\
\hline
\end{tabular}




\begin{tabular}{|c|c|c|c|c|}
\hline Hawaii & $3598(2.0)$ & $2082(1.1)$ & $1516(0.8)$ & \\
\hline Lowa & $8617(4.7)$ & $4503(2.4)$ & $4114(2.2)$ & \\
\hline New Mexico & $3768(2.0)$ & $2115(1.1)$ & $1653(0.9)$ & \\
\hline Atlanta (Metropolitan) & $5582(3.0)$ & $3005(1.6)$ & $2577(1.4)$ & \\
\hline San Jose-Monterey & $4544(2.5)$ & $2446(1.3)$ & $2098(1.1)$ & \\
\hline Los Angeles & $19336(10.5)$ & $10349(5.6)$ & $8987(4.9)$ & \\
\hline California excluding SF/SJM/LA & $40031(21.7)$ & $21667(11.8)$ & $18364(10.0)$ & \\
\hline Kentucky & $12592(6.8)$ & $6852(3.7)$ & $5740(3.1)$ & \\
\hline Louisiana & $11946(6.5)$ & $6587(3.6)$ & $5359(2.9)$ & \\
\hline New Jersey & $20202(11.0)$ & $10437(5.7)$ & $9765(5.3)$ & \\
\hline Greater Georgia & $13657(7.4)$ & $7451(4.0)$ & $6206(3.4)$ & \\
\hline Utah & $3412(1.9)$ & $1894(1.0)$ & $1518(0.8)$ & \\
\hline Seattle (Puget Sound) & $8849(4.8)$ & $4780(2.6)$ & $4069(2.2)$ & \\
\hline Rural Georgia & $367(0.2)$ & $217(0.1)$ & $150(0.1)$ & \\
\hline Marital Status no. (\%) & & & & 0.000 \\
\hline single (never married) & $29172(15.8)$ & $16389(8.9)$ & $12783(6.9)$ & \\
\hline Married (including common law) & $104562(56.7)$ & $65531(35.6)$ & $39031(21.2)$ & \\
\hline Widowed & $32370(17.6)$ & $8026(4.4)$ & $24344(13.2)$ & \\
\hline Divorced & $18218(9.9)$ & $8856(4.8)$ & $9362(5.1)$ & \\
\hline Insurance status no. (\%) & & & & 0.000 \\
\hline Insured & $155881(84.6)$ & $84222(45.7)$ & $71659(38.9)$ & \\
\hline Any Medicaid & $22111(12.0)$ & $10890(5.9)$ & $11221(6.1)$ & \\
\hline Uninsured & $6330(3.4)$ & $3690(2.0)$ & $2640(1.4)$ & \\
\hline $\begin{array}{l}\text { County-level income at time of } \\
\text { diagnosis no. }(\%)^{\mathrm{a}}\end{array}$ & & & & 0.000 \\
\hline Quartile $1(<$ US $\$ 48,540)$ & $46193(25.1)$ & $25152(13.6)$ & $21041(11.4)$ & \\
\hline Quartile 2 (US $\$ 48,550-55,870$ ) & $47580(25.8)$ & $25568(13.9)$ & $22012(11.9)$ & \\
\hline Quartile 3 (US $\$ 55,870-68,520$ ) & $44519(24.2)$ & $23839(12.9)$ & $20680(11.2)$ & \\
\hline Quartile 4 (> US $\$ 68,520)$ & $46030(25.0)$ & $24243(13.2)$ & $21787(11.8)$ & \\
\hline County-level education no. $(\%)^{b}$ & & & & 0.000 \\
\hline Quartile $1(<21.06 \%)$ & $45174(25.1)$ & $25536(13.9)$ & $20638(11.2)$ & \\
\hline Quartile $2(21.07-29.91 \%)$ & $55138(29.9)$ & $29494(16.0)$ & $25644(13.9)$ & \\
\hline Quartile 3 (29.92-35.57\%) & $37113(20.1)$ & $19722(10.7)$ & $17391(9.4)$ & \\
\hline Quartile $4(>35.57 \%)$ & 45897 (24.9) & $24050(13.0)$ & $21847(11.9)$ & \\
\hline
\end{tabular}

Abbreviations: CRC indicates colorectal cancer; SEER, Surveillance, Epidemiology, and End Results.

${ }^{a}$ Counties ranked by median household income in US $\$ 10$.

${ }^{\mathrm{b} C o u n t i e s ~ r a n k e d ~ b y ~ t h e ~ p e r c e n t a g e ~ o f ~ a d u l t ~ i n d i v i d u a l s ~ w i t h ~ a ~ B a c h e l o r ' s ~ d e g r e e . ~}$

of insurance is associated with an elevated risk of latestage diagnosis and a decreased likelihood of undergoing screening and receiving treatment following a diagnosis [17]. What's more, in a 2011 survey, nearly one third of US physicians were unwilling to accept new patients with Medicaid insurance. It might cause inappropriate treatment for those Medicaid cancer patients [18]. Until ways are found to provide health insurance to all citizens, it seems likely that the uninsured will continue to suffer poor health outcomes.

We found that the Non-Hispanic Black patients have the worst prognosis compared with the other races. Our findings are consistent with the previous studies. Actually, a number of researches have suggested that the higher mortality rate of Black colorectal cancer patients might be in part due to more invasive tumors and more advanced stage [19-21], treatment strategies variance [22-25], chemotherapy resistance [26], and screening and postsurgery surveillance. Besides, some studies highlighted that racial differences in CRC incidence and mortality could be attributed more to differences in use of healthcare than biological disparities [27].

Our study had some limitations. Firstly, the lifestyle information, comorbidities, screening and surveillance tests, or receipt of chemotherapy are not included in SEER database. Secondly, the insurance status recorded at the time of diagnosis could have changed over the follow-up period. Thirdly, some cases are not included in the cohort which may affect the generalizability to the population. Moreover, we were not able to retrieve individual-level 


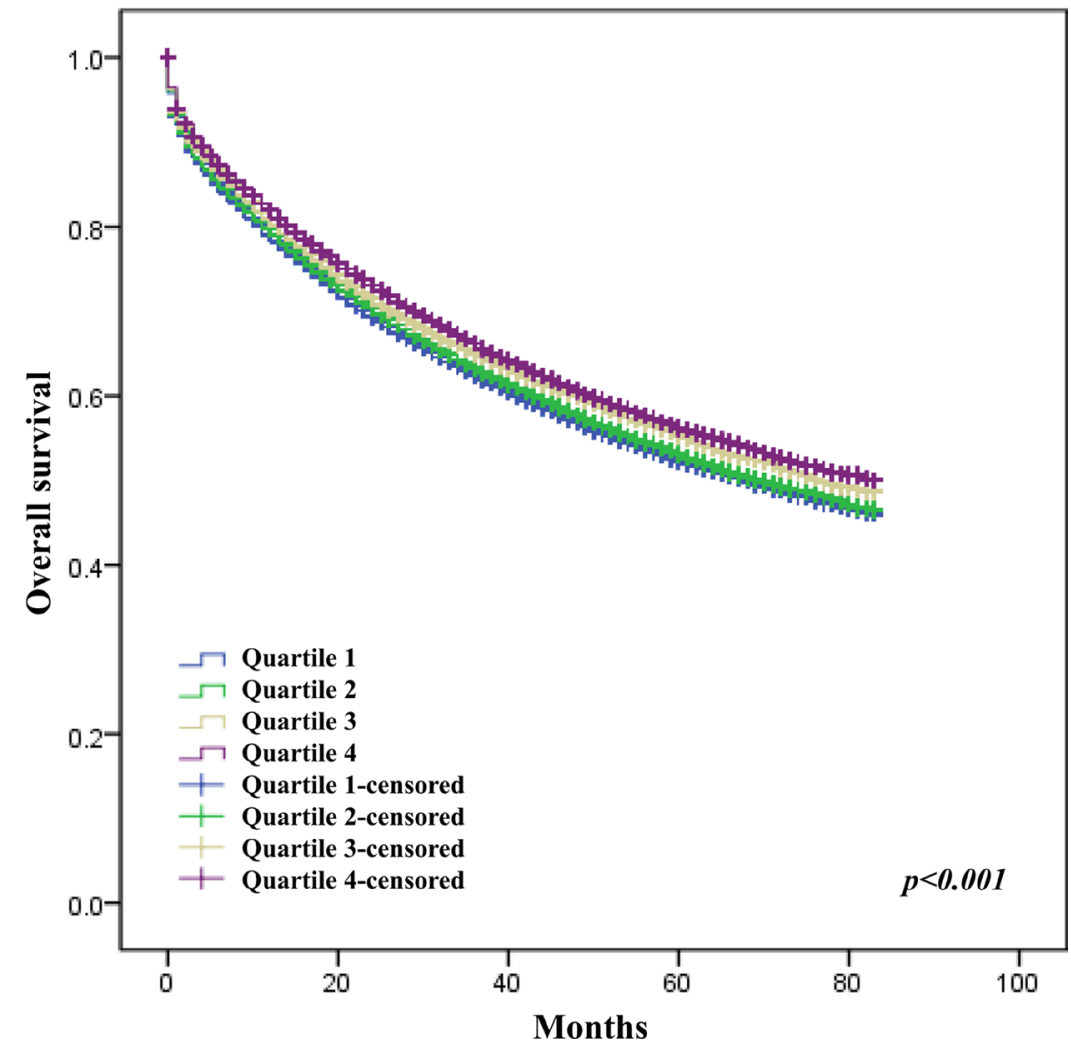

Figure 1: Effect of education level on survival of CRC patients.

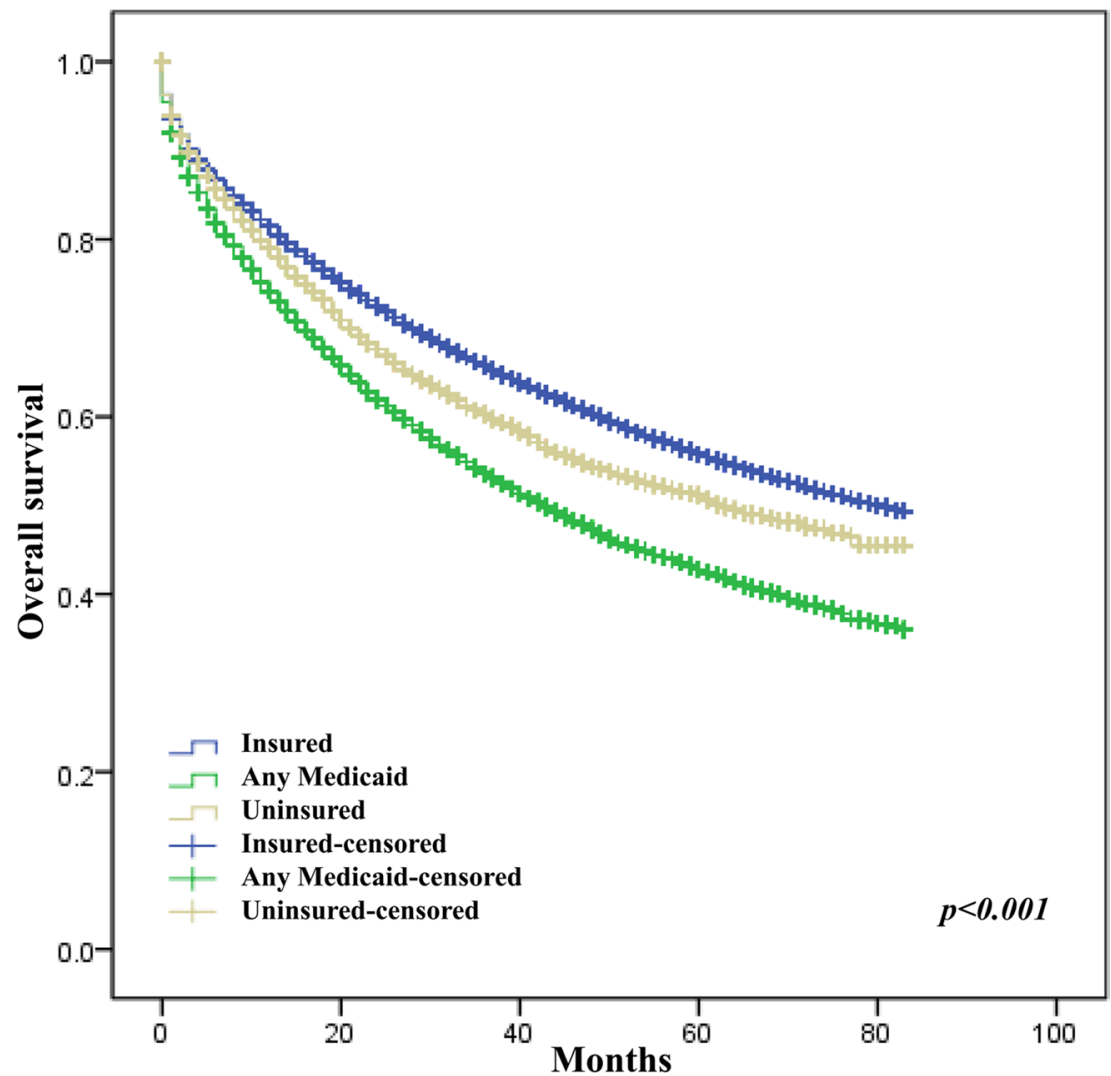

Figure 2: Effect of insurance on survival of CRC patients. 


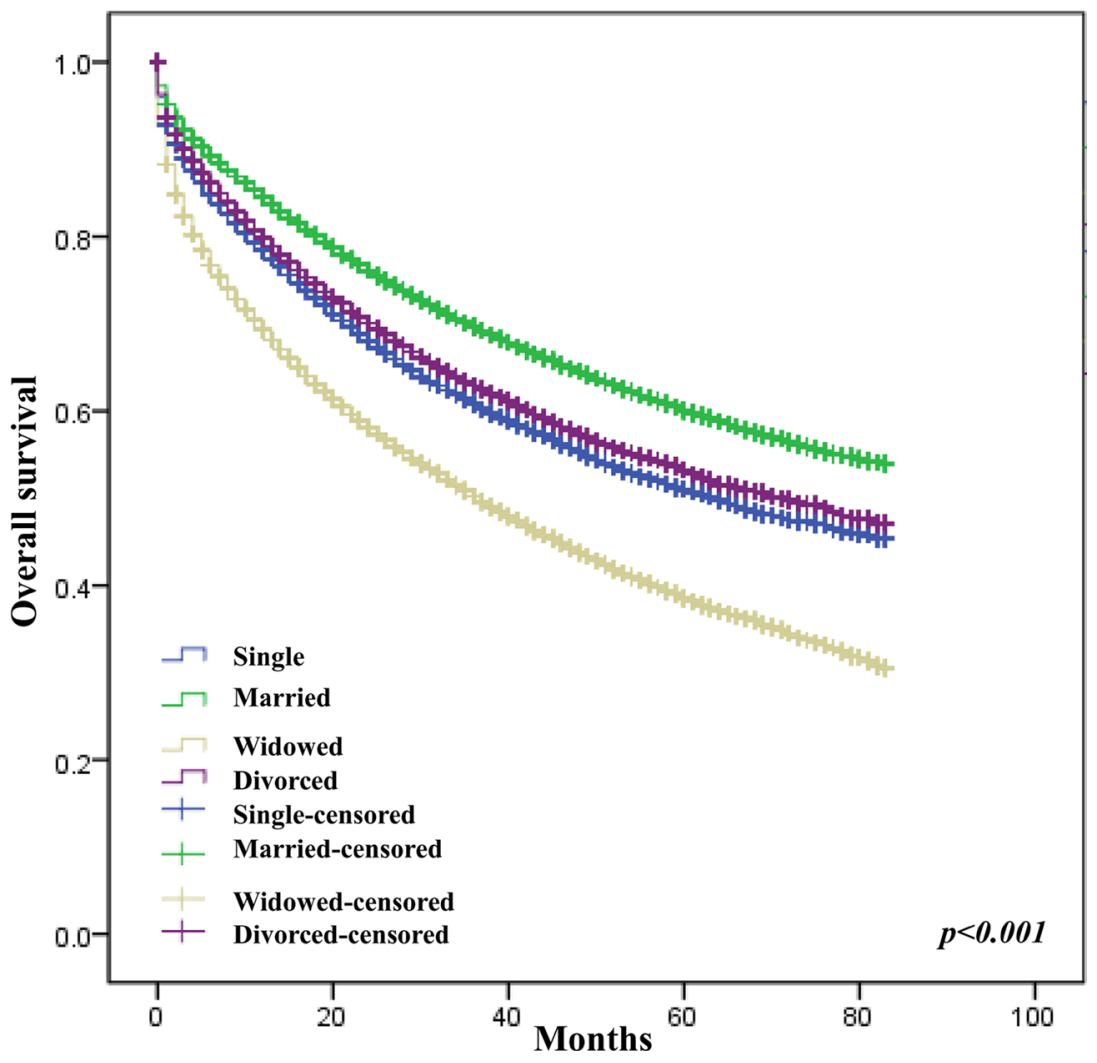

Figure 3: Effect of marital status on survival of CRC patients.

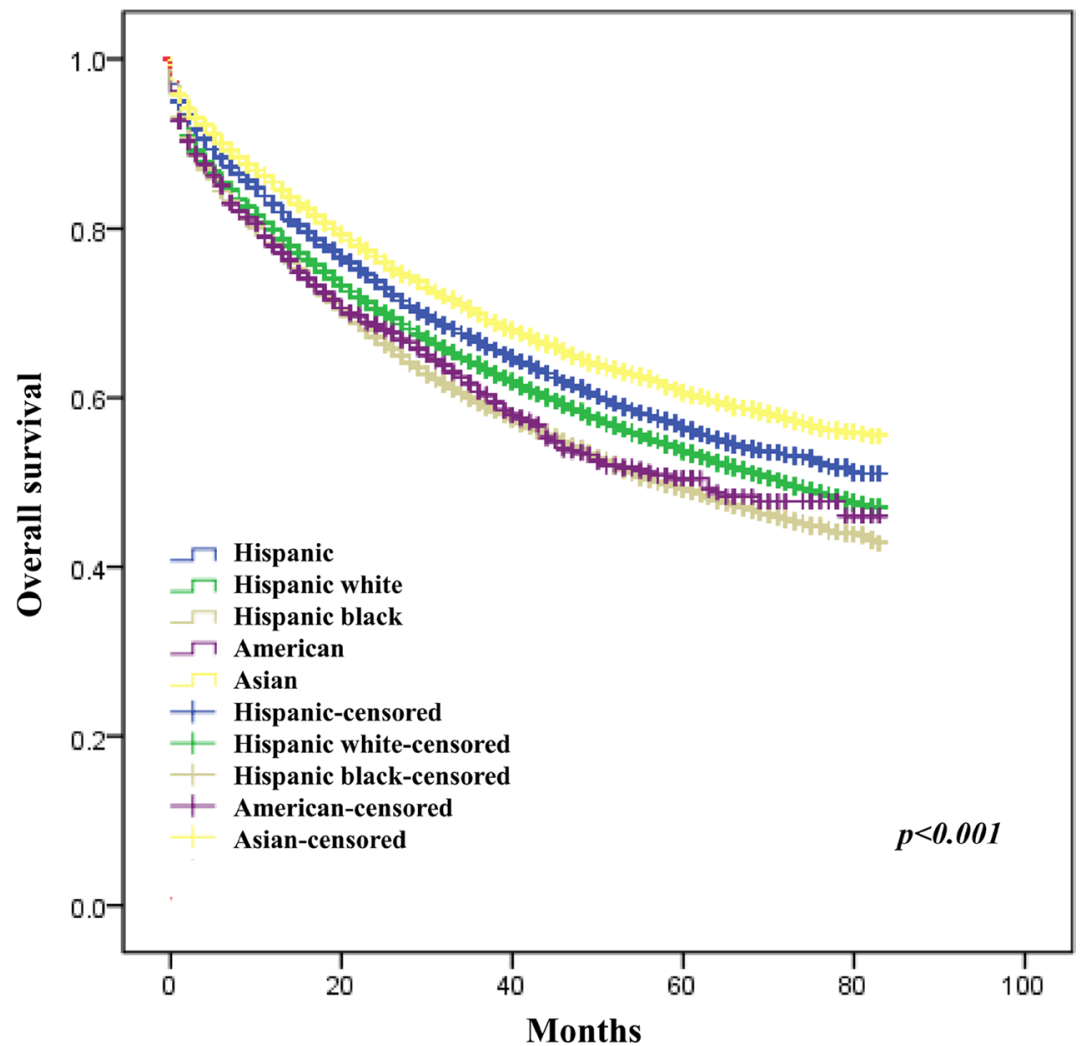

Figure 4: Effect of race on survival of CRC patients. 
Table 2: Factors associated with OS of patients with CRC at the time of diagnosis in the US SEER, 2007 to 2012

\begin{tabular}{|c|c|c|}
\hline Factors & $\begin{array}{c}\text { Crude } \\
\text { HR }(95 \% \text { CI })\end{array}$ & $\begin{array}{l}\text { Multivariable } \\
\text { HR (95\% CI) }\end{array}$ \\
\hline Female sex & $0.96(0.94-0.97)$ & $0.83(0.81-0.84)$ \\
\hline \multicolumn{3}{|l|}{ Age } \\
\hline$<45$ & 1.00 (reference) & 1.00 (reference) \\
\hline $45-59$ & $0.99(0.95-1.04)$ & $1.16(1.11-1.21)$ \\
\hline $60-74$ & $1.33(1.28-1.38)$ & $1.72(1.62-1.80)$ \\
\hline$\geq 75$ & $2.63(2.53-2.74)$ & $3.71(3.56-3.87)$ \\
\hline \multicolumn{3}{|l|}{ Site } \\
\hline Rectum VS Colon & $0.90(0.88-0.91)$ & $0.97(0.95-0.99)$ \\
\hline Left VS Right colon & $0.95(0.92-0.98)$ & $0.98(0.95-1.01)$ \\
\hline \multicolumn{3}{|l|}{ Differentiation } \\
\hline Well & 1.00 (reference) & 1.00 (reference) \\
\hline Moderate & $1.33(1.29-1.37)$ & $1.05(0.99-1.12)$ \\
\hline Poor & $2.34(2.26-2.43)$ & $1.42(1.33-1.52)$ \\
\hline Undifferentiation & $2.64(2.50-2.80)$ & $1.63(1.48-1.80)$ \\
\hline \multicolumn{3}{|l|}{ AJCC stage } \\
\hline I & 1.00 (reference) & 1.00 (reference) \\
\hline II & $1.37(1.33-1.40)$ & $1.17(1.12-1.23)$ \\
\hline III & $1.72(1.67-1.76)$ & $1.85(1.76-1.94)$ \\
\hline IV & $7.29(7.11-7.47)$ & $7.82(7.46-8.21)$ \\
\hline \multicolumn{3}{|l|}{ Race } \\
\hline Hispanic & 1.00 (reference) & 1.00 (reference) \\
\hline Non-Hispanic White & $1.13(1.10-1.16)$ & $1.06(1.03-1.09)$ \\
\hline Non-Hispanic Black & $1.27(1.23-1.32)$ & $1.20(1.15-1.24)$ \\
\hline Non-Hispanic American/Alaska native & $1.23(1.12-1.36)$ & $1.12(1.00-1.26)$ \\
\hline Non-Hispanic Asian or Pacific Islander & $0.87(0.84-0.91)$ & $0.89(0.86-0.93)$ \\
\hline Other & $0.35(0.28-0.44)$ & $0.53(0.42-0.66)$ \\
\hline \multicolumn{3}{|l|}{ Registry } \\
\hline San Francisco-Oakland SMSA & 1.00 (reference) & 1.00 (reference) \\
\hline Connecticut & $1.07(1.02-1.13)$ & $0.91(0.86-0.96)$ \\
\hline Detroit (Metropolitan) & $1.25(1.20-1.32)$ & $1.03(0.97-1.09)$ \\
\hline Hawaii & $0.93(0.87-0.997)$ & $1.01(0.94-1.09)$ \\
\hline Lowa & $1.13(1.07-1.18)$ & $0.94(0.89-0.99)$ \\
\hline New Mexico & $1.12(1.05-1.19)$ & $0.99(0.92-1.06)$ \\
\hline Atlanta (Metropolitan) & $1.06(1.00-1.12)$ & $1.04(0.97-1.10)$ \\
\hline San Jose-Monterey & $0.93(0.87-0.99)$ & $0.92(0.86-0.98)$ \\
\hline Los Angeles & $1.07(1.03-1.12)$ & $0.88(0.83-0.93)$ \\
\hline California excluding SF/SJM/LA & $1.07(1.03-1.11)$ & $0.97(0.93-1.01)$ \\
\hline Kentucky & $1.20(1.15-1.25)$ & $1.04(0.99-1.10)$ \\
\hline Louisiana & $1.15(1.10-1.20)$ & $0.97(0.92-1.03)$ \\
\hline New Jersey & $1.21(1.16-1.26)$ & $0.99(0.95-1.03)$ \\
\hline Greater Georgia & $1.12(1.08-1.18)$ & $1.01(0.96-1.06)$ \\
\hline Utah & $0.996(0.93-1.07)$ & $1.05(0.98-1.13)$ \\
\hline Seattle (Puget Sound) & $1.01(0.96-1.06)$ & $0.94(0.89-0.99)$ \\
\hline Rural Georgia & $1.21(1.02-1.44)$ & $1.02(0.86-1.21)$ \\
\hline \multicolumn{3}{|l|}{ Marital Status } \\
\hline single(never married) & 1.00 (reference) & 1.00 (reference) \\
\hline Married(including common law) & $0.74(0.72-0.75)$ & $0.74(0.73-0.76)$ \\
\hline
\end{tabular}


Widowed

Divorced

Insurance status

Insured

Any Medicaid

Uninsured

County-level income at time of diagnosis

Quartile 1 (< US \$48,540)

Quartile 2 (US \$48,550-55,870)

Quartile 3 (US \$55,870-68,520)

Quartile 4 (> US \$68,520)

County-level education

Quartile $1(<21.06 \%)$

Quartile 2 (21.07-29.91\%)

Quartile 3 (29.92-35.57\%)

Quartile 4 (>35.57\%)
$1.44(1.40-1.47)$

$0.93(0.90-0.96)$

1.04 (1.01-1.07)

$0.92(0.89-0.95)$

1.00 (reference)

1.00 (reference)

1.45 (1.42-1.48)

$1.36(1.33-1.40)$

$1.17(1.12-1.22)$

$1.32(1.26-1.38)$

1.00 (reference)

1.00 (reference)

$0.92(0.91-0.94)$

1.03 (0.998-1.06)

$0.91(0.89-0.93)$

$0.995(0.97-1.03)$

$0.86(0.84-0.87)$

$1.00(0.96-1.05)$

1.00 (reference)

1.00 (reference)

0.97 (0.95-0.99)

$0.96(0.93-0.98)$

$0.92(0.90-0.94)$

$0.92(0.89-0.94)$

$0.88(0.86-0.90)$

$0.87(0.84-0.90)$

Abbreviations: CRC indicates colorectal cancer; SEER, Surveillance, Epidemiology, and End Results.

${ }^{a}$ Counties ranked by median household income in US \$10.

${ }^{b}$ Counties ranked by the percentage of adult individuals with a Bachelor's degree.

SES factors from the SEER Limited-Use data. Census county-level measures are imperfect indicators of individual-level SES [28], and accordingly, our results may be somewhat biased by residual confounding. Lastly, some unknown or unidentified confounders could also impact the analysis.
Despite these potential limitations, our data confirmed that unmarried, Medicaid, lower education level and Non-Hispanic Black CRC patients are at a greater risk of mortality. The physicians, government and health care systems should provide specific cares and interventions for these patients to improve the survival of these patients.

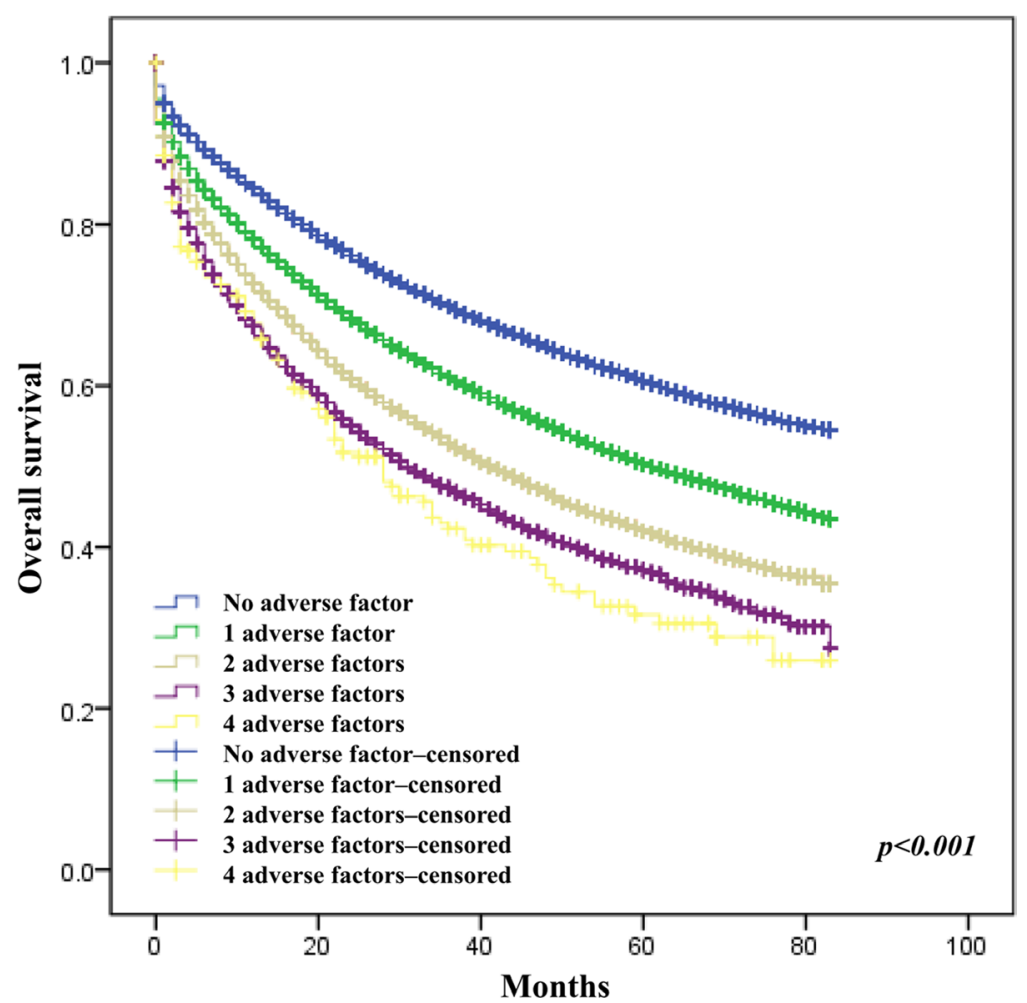

Figure 5: Survival according to the number of adverse SES factors: being widowed, uninsured, Non-Hispanic Black or lowest education level. 
Table 3: Survival analysis according to number of adverse socioeconomic factors-adjusted for age, sex, differentiation, site and stage

\begin{tabular}{|c|c|c|c|c|c|c|c|c|c|c|}
\hline & \multicolumn{2}{|c|}{ No adverse factors } & \multicolumn{2}{|c|}{1 Adverse factor } & \multicolumn{2}{|c|}{2 Adverse factors } & \multicolumn{2}{|c|}{3 Adverse factors } & \multicolumn{2}{|c|}{4 Adverse factors } \\
\hline & NO. $(\%)$ & HR $(95 \% \mathrm{CI})$ & NO. (\%) & HR $(95 \% \mathrm{CI})$ & NO. (\%) & HR $(95 \% \mathrm{CI})$ & NO. (\%) & HR $(95 \% \mathrm{CI})$ & NO. (\%) & HR $(95 \% \mathrm{CI})$ \\
\hline Female Sex & $36035(40.1)$ & $0.85(0.81-0.89)$ & $34886(49.5)$ & $0.87(0.83-0.90)$ & $12329(59.5)$ & $0.78(0.72-0.84)$ & $2081(68.8)$ & $0.80(0.67-0.98)$ & $189(82.9)$ & $0.52(0.18-1.50)$ \\
\hline $\begin{array}{l}\text { Median age } \\
\text { (range), } y\end{array}$ & $65(20-102)$ & & $69(20-107)$ & & $71(20-108)$ & & $72(21-101)$ & & $75(49-105)$ & \\
\hline$<45$ & $6177(6.9)$ & 1 (reference) & $3499(5.0)$ & 1 (reference) & $979(4.7)$ & 1 (reference) & $116(3.8)$ & 1 (reference) & 0 & - \\
\hline $45-59$ & $26129(29.1)$ & $1.21(1.06-1.38)$ & $16167(22.9)$ & $1.23(1.05-1.44)$ & 4415 (21.3) & $1.27(0.99-1.64)$ & $535(17.7)$ & $0.78(0.45-1.34)$ & $24(10.5)$ & 1 (reference) \\
\hline $60-74$ & $33943(37.8)$ & $1.70(1.49-1.93)$ & $24633(35.0)$ & $1.61(1.39-1.87)$ & $6776(32.7)$ & $1.87(1.46-2.38)$ & $1038(34.3)$ & $1.04(0.63-1.74)$ & $84(36.8)$ & $1.24(0.32-4.79)$ \\
\hline$\geq 75$ & $23660(26.3)$ & $3.60(3.17-4.09)$ & $26152(37.1)$ & $3.29(2.84-3.82)$ & 8539 (41.2) & $3.44(2.71-4.38)$ & $1336(44.2)$ & $1.72(1.04-2.83)$ & $120(52.6)$ & $2.26(0.64-8.95)$ \\
\hline \multicolumn{11}{|l|}{ Differentiation } \\
\hline Well & $7676(8.5)$ & 1 (reference) & $5794(8.2)$ & 1 (reference) & $1686(8.1)$ & 1 (reference) & $255(8.4)$ & 1.00 (reference) & $25(11.0)$ & 1 (reference) \\
\hline Moderate & $55574(61.8)$ & $1.04(0.94-1.15)$ & $43373(61.6)$ & $1.02(0.93-1.12)$ & 12764 (61.6) & $1.14(0.99-1.33)$ & $1820(60.2)$ & $1.12(0.78-1.61)$ & $151(66.2)$ & $1.28(0.37-4.40)$ \\
\hline Poor & $13270(14.8)$ & $1.51(1.36-1.67)$ & $10177(14.4)$ & $1.38(1.25-1.53)$ & $2777(13.4)$ & $1.51(1.28-1.78)$ & $423(14.0)$ & $1.55(1.05-2.31)$ & $23(10.1)$ & $3.06(0.06-15.58)$ \\
\hline $\begin{array}{c}\text { Un- } \\
\text { differentiation }\end{array}$ & $1616(1.8)$ & $1.65(1.42-1.93)$ & $1543(2.2)$ & $1.62(1.41-1.87)$ & $445(2.1)$ & $2.04(1.62-2.56)$ & $55(1.8)$ & $1.45(0.78-2.69)$ & $2(0.9)$ & $4.03(0.29-56.47)$ \\
\hline \multicolumn{11}{|l|}{ Site } \\
\hline Colon & $61730(68.7)$ & & $49495(70.3)$ & & $14801(71.5)$ & & $2157(71.3)$ & & $159(69.7)$ & \\
\hline Right colon & $17782(19.8)$ & & $15676(22.3)$ & & $4742(22.9)$ & & 661 (21.9) & & $51(22.4)$ & \\
\hline Left colon & $6922(7.7)$ & $0.94(0.89-0.99)$ & $5660(8.0)$ & $0.95(0.90-0.999)$ & $1842(8.9)$ & $1.07(0.98-1.16)$ & $315(10.4)$ & $0.95(0.78-1.15)$ & $23(10.1)$ & $2.20(0.96-4.28)$ \\
\hline Rectum & $24918(27.7)$ & $0.97(0.94-0.99)$ & $18067(25.6)$ & $0.99(0.96-1.02)$ & $4939(23.8)$ & $0.97(0.93-1.02)$ & $696(23.0)$ & $0.91(0.80-1.03)$ & $56(24.6)$ & $1.02(0.66-1.58)$ \\
\hline $\begin{array}{c}\text { Large } \\
\text { intestine,NOS }\end{array}$ & $2614(2.9)$ & & $2498(3.5)$ & & $873(4.2)$ & & $159(5.3)$ & & $13(5.7)$ & \\
\hline \multicolumn{11}{|l|}{$\begin{array}{l}\text { AJCC stage, } \\
\text { no. }(\%)\end{array}$} \\
\hline I & $22340(24.8)$ & 1 (reference) & $16321(23.2)$ & 1 (reference) & 4427 (21.4) & 1 (reference) & $645(21.3)$ & 1 (reference) & $55(24.1)$ & 1 (reference) \\
\hline II & $21034(23.4)$ & $1.25(1.16-1.36)$ & $17329(24.6)$ & $1.20(1.11-1.29)$ & $5052(24.4)$ & $0.98(0.86-1.11)$ & $756(25.0)$ & $0.82(0.60-1.11)$ & $52(22.8)$ & $0.35(0.12-0.99)$ \\
\hline III & $23165(25.8)$ & $1.98(1.83-2.15)$ & $17164(24.4)$ & $1.87(1.74-2.02)$ & 4813 (23.2) & $1.62(1.43-1.84)$ & $647(21.4)$ & $1.42(1.05-1.92)$ & $68(29.8)$ & $0.43(0.17-1.11)$ \\
\hline IV & $16115(17.9)$ & $9.50(8.81-10.25)$ & $13432(19.1)$ & $7.42(6.89-7.98)$ & 4405 (21.3) & $5.92(5.25-6.68)$ & $657(21.7)$ & $4.58(3.41-6.15)$ & $28(12.3)$ & $0.78(0.25-2.460)$ \\
\hline Unstaged & $7255(8.1)$ & & $6205(8.8)$ & & $2012(9.7)$ & & $320(10.6)$ & & $25(11.0)$ & \\
\hline
\end{tabular}

\section{MATERIALS AND METHODS}

\section{Patient selection in the SEER database}

Patients' data were from the Surveillance, Epidemiology, and End Results (SEER) registry program (http://seer.cancer.gov/data/citation.html). The SEER-18 includes population-based cancer populations reported in the Atlanta, Connecticut, Detroit, Hawaii, Iowa, New Mexico, San Francisco-Oakland, Seattle-Puget Sound, Utah, Los Angeles, San Jose-Monterey, Rural Georgia, Alaska Native, Greater California, Kentucky, Louisiana, New Jersey, and Greater Georgia registries, that represent approximately $28 \%$ of the population in the US. The SEER has been widely used in many cancer researches.

Using the SEER-stat software (SEER*Stat 8.3.2), we selected patients that were newly diagnosed with CRC between 2007 (the year when insurance information were available) and 2013 (the most recent year for which data were available) with single primary CRC. The exclusion criteria included that patients at diagnosis were less than 18 years old, had undefined TNM stage, developed more than one primary cancer but the CRC wasn't the first one, had unknown insurance status, marital status, income or education level, had unknown cause of death or unknown survival months. The 7th AJCC staging edition was adopted to redefine stage I, II, III, and IV. Patients with AJCC stage 0 disease were also excluded.

\section{Socioeconomic factors analysis}

The following factors were included and analyzed: age at the time of diagnosis (continuous), gender, race/ ethnicity (Hispanic, non-Hispanic white, non-Hispanic black, Non-Hispanic American/Alaska native, NonHispanic Asian or Pacific Islander and other), SEER registry, marital status (married, divorced, single, or widowed), insurance status (any Medicaid, insured, or uninsured), median household income (in quartiles), percentage of adult individuals with at least a Bachelor's degree within the county of residence (in quartiles), follow-up time, and vital status (alive or dead).

\section{Statistical analysis}

The $\chi 2$ test was adopted to analyze individuals' basic characteristics appropriately. Cox proportional hazards models were used to calculate the overall survival of CRC patients with the hazard ratio (HR) and corresponding $95 \%$ confidence interval $(95 \% \mathrm{CI})$. The confounding factors including gender, age, education (in quartiles), household income (in quartiles), and race/ethnicity were 
adjusted. Survival plots were generated using the KaplanMeier method. A 2-sided $p$ value 0.05 was considered to be statistically significant. All analyses were performed using the statistical software package SPSS for Windows, version 17 (SPSS Inc., Chicago, IL, USA).

\section{Abbrivations}

Colorectal cancer, CRC; socioeconomic, status SES; hazard ratio, HR; confidence interval, CI; Surveillance, Epidemiology, and End Results, SEER; overall survival, OS;

\section{Authors' contributions}

ZQ, WGY and WXS conceived of and designed the study. ZQ and WYF wrote the manuscript. HHQ, HR, $\mathrm{XL}$ and CYG performed the analyses. LER prepared all figures and tables. All authors reviewed the manuscript.

\section{ACKNOWLEDGMENTS}

We thank engineer Dandan Qin for the help of statistical analysis.

\section{CONFLICTS OF INTEREST}

None of the authors have any conflicts of interest to declare.

\section{FUNDING}

This research was funded by Harbin technology innovation fund for young experts (WGY), Natural Science Foundation of Heilongjiang Province (D201205) and Innovation fund for young of Harbin Medical University (2017LCZX66).

\section{REFERENCES}

1. Siegel RL, Miller KD, Jemal A. Cancer statistics. CA Cancer J Clin. 2017; 67:7-30.

2. Jin JJ, Wang W, Dai FX, Long ZW, Cai H, Liu XW, Zhou Y, Huang H, Wang YN. Marital status and survival in patients with gastric cancer. Cancer Med. 2016; 5:1821-1829.

3. Wang XD, Qian JJ, Bai DS, Li ZN, Jiang GQ, Yao J. Marital status independently predicts pancreatic cancer survival in patients treated with surgical resection: An analysis of the seer database. Oncotarget. 2016; 7:24880-24887. https:// doi.org/10.18632/oncotarget.8467.

4. Li Q, Gan L, Liang L, Li X, Cai S. The influence of marital status on stage at diagnosis and survival of patients with colorectal cancer. Oncotarget. 2015; 6:7339-7347. https:// doi.org/10.18632/oncotarget.3129.

5. Aizer AA, Chen MH, Mccarthy EP, Mendu ML, Koo S, Wilhite TJ, Graham PL, Choueiri TK, Hoffman KE,
Martin NE, Hu JC, Nguyen PL. Marital status and survival in patients with cancer. J Clin Oncol. 2013; 31:3869-3876.

6. Shi RL, Qu N, Lu ZW, Liao T, Gao Y, Ji QH. The impact of marital status at diagnosis on cancer survival in patients with differentiated thyroid cancer. Cancer Med. 2016; 5: 2145-2154.

7. Thong MS, Kaptein AA, Krediet RT, Boeschoten EW, Dekker FW. Social support predicts survival in dialysis patients. Nephrol Dial Transplant. 2007; 22:845-850.

8. Goldzweig G, Andritsch E, Hubert A, Brenner B, Walach N, Perry S, Baider L. Psychological distress among male patients and male spouses: What do oncologists need to know? Ann Oncol. 2010; 21:877-883.

9. Shin KJ, Lee YJ, Yang YR, Park S, Suh PG, Follo MY, Cocco L, Ryu SH. Molecular mechanisms underlying psychological stress and cancer. Curr Pharm Des. 2016; 22:2389-2402.

10. Chagpar R, Xing Y, Chiang YJ, Feig BW, Chang GJ, You YN, Cormier JN. Adherence to stage-specific treatment guidelines for patients with colon cancer. J Clin Oncol. 2012; 30:972-979.

11. Serra-Rexach JA, Jimenez AB, Garcia-Alhambra MA, Pla R, Vidan M, Rodriguez P, Ortiz J, Garcia-Alfonso P, Martin M. Differences in the therapeutic approach to colorectal cancer in young and elderly patients. Oncologist. 2012; 17:1277-1285.

12. Van Der Geest KS, Abdulahad WH, Tete SM, Lorencetti PG, Horst G, Bos NA, Kroesen BJ, Brouwer E, Boots AM. Aging disturbs the balance between effector and regulatory cd4+ t cells. Exp Gerontol. 2014; 60:190-196.

13. Weiner AB, Conti RM, Eggener SE. National economic conditions and patient insurance status predict prostate cancer diagnosis rates and management decisions. J Urol. 2016; 195:1383-1389.

14. Bibbins-Domingo K, Grossman DC, Curry SJ, Davidson KW, Epling JW Jr, Garcia FA, Gillman MW, Harper DM, Kemper AR, Krist AH, Kurth AE, Landefeld CS, Mangione $\mathrm{CM}$, et al, and US Preventive Services Task Force. Screening for colorectal cancer: US Preventive Services Task Force recommendation statement. JAMA. 2016; 315:2564-2575.

15. Sung JJ, Ng SC, Chan FK, Chiu HM, Kim HS, Matsuda T, Ng SS, Lau JY, Zheng S, Adler S, Reddy N, Yeoh KG, Tsoi KK, et al. An updated asia pacific consensus recommendations on colorectal cancer screening. Gut. 2015; 64:121-132.

16. Grant SR, Walker GV, Guadagnolo BA, Koshy M, Mahmood U. A brighter future? The impact of insurance and socioeconomic status on cancer outcomes in the USA: A review. Future Oncol. 2016; 12:1507-1515.

17. Robbins AS, Siegel RL, Jemal A. Racial disparities in stagespecific colorectal cancer mortality rates from 1985 to 2008 . J Clin Oncol. 2012; 30:401-405.

18. Decker SL. In 2011 nearly one-third of physicians said they would not accept new medicaid patients, but rising fees may help. Health Aff (Millwood). 2012; 31:1673-1679. 
19. Thomas CR Jr, Jarosz R, Evans N. Racial differences in the anatomical distribution of colon cancer. Arch Surg. 1992; 127:1241-1245.

20. Gray KD, Ballard BR, Washington MK, Belue R, Grady WM, Chapman WC, Stain SC. Do adverse histopathologic findings in colorectal cancer patients explain disparate outcomes? J Natl Med Assoc. 2006; 98:348-351.

21. Tawk R, Abner A, Ashford A, Brown CP. Differences in colorectal cancer outcomes by race and insurance. Int $\mathrm{J}$ Environ Res Public Health. 2016; 13:ijerph13010048.

22. Moses KA, Orom H, Brasel A, Gaddy J, Underwood W 3rd. Racial/ethnic disparity in treatment for prostate cancer: Does cancer severity matter. Urology. 2016.

23. Schrag D, Cramer LD, Bach PB, Begg CB. Age and adjuvant chemotherapy use after surgery for stage iii colon cancer. J Natl Cancer Inst. 2001; 93:850-857.

24. Ayanian JZ, Zaslavsky AM, Fuchs CS, Guadagnoli E, Creech CM, Cress RD, O'Connor LC, West DW, Allen ME, Wolf RE, Wright WE. Use of adjuvant chemotherapy and radiation therapy for colorectal cancer in a population-based cohort. J Clin Oncol. 2003; 21:1293-1300.
25. Baldwin LM, Dobie SA, Billingsley K, Cai Y, Wright GE, Dominitz JA, Barlow W, Warren JL, Taplin SH. Explaining black-white differences in receipt of recommended colon cancer treatment. J Natl Cancer Inst. 2005; 97:1211-1220.

26. Polite BN, Dignam JJ, Olopade OI. Colorectal cancer model of health disparities: Understanding mortality differences in minority populations. J Clin Oncol. 2006; 24:2179-2187.

27. Laiyemo AO, Doubeni C, Pinsky PF, Doria-Rose VP, Bresalier R, Lamerato LE, Crawford ED, Kvale P, Fouad M, Hickey T, Riley T, Weissfeld J, Schoen RE, et al. Race and colorectal cancer disparities: Healthcare utilization vs different cancer susceptibilities. J Natl Cancer Inst. 2010; 102:538-546.

28. Weng Y, Korte JE. Racial disparities in being recommended to surgery for oral and oropharyngeal cancer in the united states. Community Dent Oral Epidemiol. 2012; 40:80-88. 